International Journal of Electrical and Power Engineering 4 (3): 169-176, 2010

ISSN: 1990-7958

(C) Medwell Journals, 2010

\title{
Using A New Hybrid Method for Optimal Placement of Unified Power Flow Controller in Power System
}

\author{
${ }^{1}$ R. Jahani, ${ }^{2}$ A. Safari, ${ }^{1}$ J. Olamaei and ${ }^{2}$ H.A. Shayanfar \\ ${ }^{1}$ Department of Electrical Engineering, Islamic Azad University, South Tehran Branch, Tehran, Iran \\ ${ }^{2}$ Department of Electrical Engineering, \\ Center of Excellence for Power System Automation and Operation, \\ Iran University of Science and Technology, Tehran, Iran
}

\begin{abstract}
This study presents a hybrid genetic and particle swarm optimization algorithms to seek the optimal number and location of FACTS devices in a power system. Unified Power Flow Controller (UPFC) has great flexibility that can control the active and reactive power flow and bus voltages, simultaneously. Decoupled model of the UPFC is applied to maximize the system loadability subject to the transmission line capacity limits and specified voltage level. Optimal placement of UPFC in power system by Hybrid of GA and PSO Algorithm (HGAPSO) algorithm, it leads to a flat voltage profile and increased stability and capacity of the power transmission in lines. At the end, the proof is performed by simulating and testing the 14 bus network and placement of the UPFC appropriately. The results show that the steady state performance of power system can be effectively enhanced due to the optimal location and parameters of the UPFC.
\end{abstract}

Key words: Hybrid GA, PSO algorithm, UPFC, power flow, optimal location, Iran

\section{INTRODUCTION}

Recently, the steady state performance of power system has become a matter of grave concern in system operation and planning. As the power system becomes more complex and more heavily loaded, it can be operated in unstable or insecure situations like the cascading thermal overloads, the frequency and voltage collapse. For a secure operation of the power system, it is essential to maintain the required level of security margin (Hao et al., 2004; Hingorani and Gyugyi, 1999; Singh and David, 2001). Then, power system controllability is required in order to utilize the available network capacitance adequately. The development of FACTS devices based on the advance of semiconductor technology opens up new opportunities for controlling the load flow and extending the loadability of the available transmission network. The UPFC is one of the family members of FACTS devices for load flow control since, it can either simultaneously or selectively control the active and reactive power flow along the lines (Nabavi-Niaki and Iravani, 1996; Noroozian et al., 1997). Several studies have been published about finding the optimal location of the UPFC with respect to different purposes and methods (Fang and Ngan, 1999; Gerbex et al., 2001). Fang and
Ngan (1999) augmented Lagrange multiplier method is applied to determine the optimal location of the UPFC to be installed. Although, multi operating conditions can simultaneously be taken into consideration, the operating condition must be pre-assigned. Gerbex et al. (2001) provides the genetic algorithm to optimize three parameters of the multi type FACTS devices including TCSC, TCPST, TCVR and SVC: the location of the devices, their types and their values but another kind of FACTS device UPFC has not been considered.

The main objective of this study is to develop an algorithm for finding and choosing the optimal location of the UPFC in order to maximizing the system loadability while simultaneously satisfying system operating constraints including transmission line capacity and voltage level limits. The optimal location problem of a given number of FACTS is converted to an optimization problem which is solved by the hybrid genetic and particle swarm optimization algorithms that has a strong ability to find the most optimistic results (Patemi et al., 1999; Coley, 1999; Shayeghi et al., 2009; Poli et al., 2007). In the following, the main results of tests on the IEEE 14 bus power system for the proposed HGAPSO method are shown to demonstrate the effectiveness of the proposed method.

Corresponding Author: A. Safari, Department of Electrical Engineering,

Center of Excellence for Power System Automation and Operation,

Iran University of Science and Technology, Tehran, Iran 
Mathematical model of UPFC: The UPFC may be seen to consist of two voltage source converters sharing a common capacitor on their DC side and a unified control system. A simplified schematic representation together with its equivalent circuit of the UPFC is shown in Fig. 1. The UPFC allows simultaneous control of the active and reactive power flow and voltage magnitude at the UPFC terminals. Alternatively, the controller may be set to control one or more of these parameters in any combination or to control none of them (Hingorani and Gyugyi, 1999). The active power demanded by the series converter is drawn by the shunt converter from the $\mathrm{AC}$ network and supplied to bus $\mathrm{m}$ through the DC link. The output voltage of the series converter is added to the nodal voltage, say bus $\mathrm{k}$ to boost the nodal voltage at bus $\mathrm{m}$. The voltage magnitude of the output voltage $V_{C R}$ provides voltage regulation and the phase angle $\mathrm{a}_{\mathrm{CR}}$ determines the mode of power flow control (Singh and David, 2001). In addition to providing a supporting role in the active power exchange that takes place between the series converter and the $\mathrm{AC}$ system, the shunt converter may also generate or absorb reactive power in order to provide independent voltage magnitude regulation at its point of connection with the $\mathrm{AC}$ system.

The UPFC equivalent circuit consists of a shuntconnected voltage source, a series connected voltage
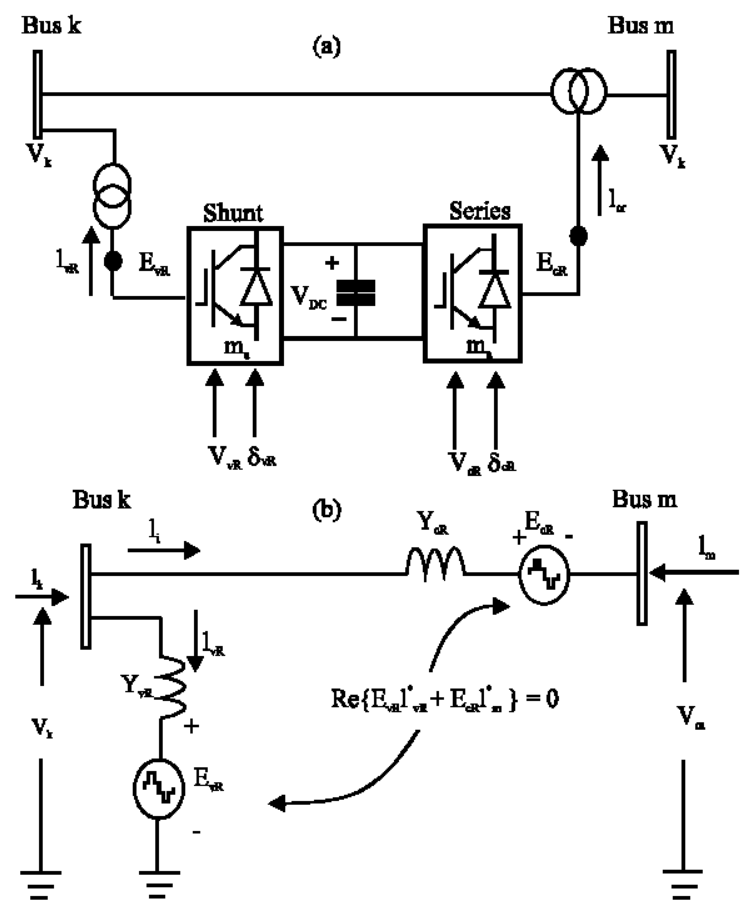

Fig. 1: Block diagram of the UPFC system: (a) Two backto-back voltage source converters (b) Equivalent circuit of a UPFC

source and an active power constraint equation which links the two voltage sources. The two voltage sources are connected to the $\mathrm{AC}$ system through inductive reactance representing the VSC transformers. In a three phase UPFC, suitable expressions for the two voltage sources and constraint equation would be given as follows:

$$
\begin{gathered}
\mathrm{E}_{\mathrm{vR}}^{\rho}=\mathrm{V}_{\mathrm{vR}}^{\rho}\left(\cos \delta_{\mathrm{vR}}^{\rho}+\mathrm{j} \sin \delta_{\mathrm{vR}}^{\rho}\right) \\
\mathrm{E}_{\mathrm{cR}}^{\rho}=\mathrm{V}_{\mathrm{cR}}^{\rho}\left(\cos \delta_{\mathrm{cR}}^{\rho}+\mathrm{j} \sin \delta_{\mathrm{cR}}^{\rho}\right) \\
\operatorname{Re}\left\{-\mathrm{E}_{\mathrm{vR}}^{\rho} I_{\mathrm{cR}}^{*_{\rho}}+\mathrm{E}_{\mathrm{vR}}^{\rho} I_{\mathrm{m}}^{*}\right\}=0
\end{gathered}
$$

Where, $\rho$ indicates phase quantities a, $\mathrm{b}$ and $\mathrm{c}$. Similar to the shunt and series voltage sources used to represent the STATCOM and the SSSC, respectively the voltage sources used in the UPFC application would also have limits.

Based on the equivalent circuit shown in Fig. 1 and assuming three phase parameters, the following transfer admittance equation can be written:

$$
\left[\begin{array}{l}
I_{k} \\
I_{m}
\end{array}\right]=\left[\begin{array}{lrrc}
\left(Y_{c R}+Y_{v R}\right) & -Y_{c R} & -Y_{c R} & -Y_{v R} \\
-Y_{c R} & Y_{c R} & Y_{c R} & 0
\end{array}\right] \text { (4) }
$$

Where all the parameters have been defined:

$$
\begin{gathered}
\mathrm{I}_{\mathrm{k}}=\left[\begin{array}{lll}
\mathrm{I}_{\mathrm{k}}^{\mathrm{a}} \angle \mathrm{Y}_{\mathrm{k}}^{\mathrm{a}} & \mathrm{I}_{\mathrm{k}}^{\mathrm{b}} \angle \mathrm{Y}_{\mathrm{k}}^{\mathrm{b}} & \mathrm{I}_{\mathrm{k}}^{\mathrm{c}} \angle \mathrm{Y}_{\mathrm{k}}^{\mathrm{c}}
\end{array}\right]^{\mathrm{t}} \\
\mathrm{V}_{\mathrm{k}}=\left[\begin{array}{lll}
\mathrm{V}_{\mathrm{k}}^{\mathrm{a}} \angle \theta_{\mathrm{k}}^{\mathrm{a}} & \mathrm{V}_{\mathrm{k}}^{\mathrm{b}} \angle \theta_{\mathrm{k}}^{\mathrm{b}} & \mathrm{V}_{\mathrm{k}}^{\mathrm{c}} \angle \theta_{\mathrm{k}}^{\mathrm{c}}
\end{array}\right]^{\mathrm{t}} \\
\mathrm{E}_{\mathrm{vR}}=\left[\begin{array}{lll}
\mathrm{V}_{\mathrm{vR}, \mathrm{K}}^{\mathrm{a}} \angle \delta_{\mathrm{vR}, \mathrm{K}}^{\mathrm{a}} & \mathrm{V}_{\mathrm{vR}, \mathrm{K}}^{\mathrm{b}} \angle \delta_{\mathrm{vR}, \mathrm{K}}^{\mathrm{b}} & \mathrm{V}_{\mathrm{vR}, \mathrm{K}}^{\mathrm{c}} \angle \delta_{\mathrm{vR}, \mathrm{K}}^{\mathrm{c}}
\end{array}\right]^{\mathrm{t}}
\end{gathered}
$$

$$
\begin{gathered}
\mathrm{I}_{\mathrm{m}}=\left[\begin{array}{lll}
\mathrm{I}_{\mathrm{m}}^{\mathrm{a}} \angle \gamma_{\mathrm{m}}^{\mathrm{a}} & \mathrm{I}_{\mathrm{m}}^{\mathrm{b}} \angle \gamma_{\mathrm{m}}^{\mathrm{b}} & \mathrm{I}_{\mathrm{m}}^{\mathrm{c}} \angle \gamma_{\mathrm{m}}^{\mathrm{c}}
\end{array}\right]^{\mathrm{t}} \\
\mathrm{V}_{\mathrm{m}}=\left[\begin{array}{lll}
\mathrm{V}_{\mathrm{m}}^{\mathrm{a}} \angle \theta_{\mathrm{m}}^{\mathrm{a}} & \mathrm{V}_{\mathrm{m}}^{\mathrm{b}} \angle \theta_{\mathrm{m}}^{\mathrm{b}} & \mathrm{V}_{\mathrm{m}}^{\mathrm{c}} \angle \theta_{\mathrm{m}}^{\mathrm{c}}
\end{array}\right]^{\mathrm{t}} \\
\mathrm{E}_{\mathrm{cR}}=\left[\begin{array}{lll}
\mathrm{V}_{\mathrm{cR}}^{\mathrm{a}} \angle \delta_{\mathrm{cR}}^{\mathrm{a}} & \mathrm{V}_{\mathrm{cR}}^{\mathrm{b}} \angle \delta_{\mathrm{cR}}^{\mathrm{b}} & \mathrm{V}_{\mathrm{cR}}^{\mathrm{c}} \angle \delta_{\mathrm{cR}}^{\mathrm{c}}
\end{array}\right]^{\mathrm{t}}
\end{gathered}
$$




$$
\mathrm{Y}_{\mathrm{CR}}=\left[\begin{array}{ccc}
\mathrm{Y}_{\mathrm{cR}, \mathrm{k}}^{\mathrm{a}} & 0 & 0 \\
0 & \mathrm{Y}_{\mathrm{cR}, \mathrm{k}}^{\mathrm{b}} & 0 \\
0 & 0 & \mathrm{Y}_{\mathrm{cR}, \mathrm{k}}^{\mathrm{c}}
\end{array}\right]
$$

An equivalent circuit consisting of two coordinated synchronous voltage sources should represent the UPFC adequately for the purpose of fundamental frequency steady-state analysis. Such an equivalent circuit is shown in Fig. 2. The synchronous voltage sources represent the fundamental Fourier series component of the switched voltage waveforms at the $\mathrm{AC}$ converter terminals of the UPFC (Hingorani and Gyugyi, 1999).

$$
\begin{aligned}
& \mathrm{E}_{\mathrm{vR}}=\mathrm{V}_{\mathrm{vR}}\left(\cos \delta_{\mathrm{vR}}+\mathrm{j} \sin \delta_{\mathrm{vR}}\right) \\
& \mathrm{E}_{\mathrm{cR}}=\mathrm{V}_{\mathrm{cR}}\left(\cos \delta_{\mathrm{cR}}+\mathrm{j} \sin \delta_{\mathrm{cR}}\right)
\end{aligned}
$$

Where, $V_{v R}$ and $\delta_{v R}$ are the controllable magnitude $\left(\mathrm{V}_{\mathrm{vR}} \min 3 \mathrm{~V}_{\mathrm{vR}} \leq \mathrm{V}_{\mathrm{vR}_{\max }}\right)$ and phase angle $\left(0 \leq \delta_{\mathrm{vR}} \leq 2 \pi\right)$ of the voltage source representing the shunt converter. The magnitude $V_{v R}$ and phase angle $\delta_{v R}$ of the voltage source representing the series converter are controlled between limits $\left(\mathrm{V}_{\mathrm{vR}} \min \frac{\mathrm{V}_{\mathrm{vR}} \leq \mathrm{V}_{\mathrm{vR}} \text { max }}{}\right)$ and $\left(0 \leq \delta_{\mathrm{vR}} \leq 2 \pi\right)$, respectively. The phase angle of the series injected voltage determines the mode of power flow control. If $\boldsymbol{\delta}_{\mathrm{vR}}$ is in phase with the nodal voltage angle $\theta_{\mathrm{k}}$, the UPFC regulates the terminal voltage. If $\delta_{\mathrm{vR}}$ is in quadature with respect to $\theta_{\mathrm{k}}$, it controls active power flow acting as a phase shifter. If $\delta_{\mathrm{vR}}$ is in quadrature with the line current angle; then it controls active power flow, acting as a variable series compensator. At any other value of $\theta_{\mathrm{k}}$, the UPFC operates as a combination of voltage regulator, variable series compensator and phase shifter. The magnitude of the series injected voltage determines the amount of power flow to be controlled. Based on the equivalent circuit shown in Fig. 2, the active and reactive power equations are obtained as:

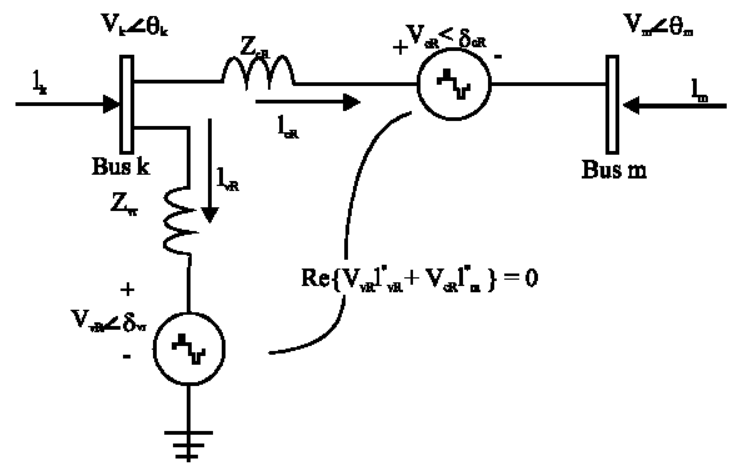

Fig. 2: Equivalent circuit of a UPFC

$$
\begin{aligned}
& P_{\mathrm{k}}=\mathrm{V}_{\mathrm{k}}^{2} \mathrm{G}_{\mathrm{kk}}+\mathrm{V}_{\mathrm{k}} \mathrm{V}_{\mathrm{m}}\left[\mathrm{G}_{\mathrm{km}} \cos \left(\theta_{\mathrm{k}}-\theta_{\mathrm{m}}\right)+\mathrm{B}_{\mathrm{km}}\right. \\
& \left.\sin \left(\theta_{\mathrm{k}}-\theta_{\mathrm{m}}\right)\right]+\mathrm{V}_{\mathrm{k}} \mathrm{V}_{\mathrm{cR}}\left[\mathrm{G}_{\mathrm{km}} \cos \left(\theta_{\mathrm{k}}-\delta_{\mathrm{cR}}\right)+\right. \\
& \left.\mathrm{B}_{\mathrm{km}} \sin \left(\theta_{\mathrm{k}}-\delta_{\mathrm{cR}}\right)\right]+\mathrm{V}_{\mathrm{k}} \mathrm{V}_{\mathrm{vR}}\left[\mathrm{G}_{\mathrm{vR}} \cos \left(\theta_{\mathrm{k}}-\delta_{\mathrm{vR}}\right)+\right. \\
& \left.\mathrm{B}_{\mathrm{vR}} \sin \left(\theta_{\mathrm{k}}-\delta_{\mathrm{vR}}\right)\right] \\
& \mathrm{Q}_{\mathrm{k}}=-\mathrm{V}_{\mathrm{k}}^{2} \mathrm{G}_{\mathrm{kk}}+\mathrm{V}_{\mathrm{k}} \mathrm{V}_{\mathrm{m}}\left[\mathrm{G}_{\mathrm{km}} \sin \left(\theta_{\mathrm{k}}-\theta_{\mathrm{m}}\right)-\right. \\
& \left.\mathrm{B}_{\mathrm{km}} \cos \left(\theta_{\mathrm{k}}-\theta_{\mathrm{m}}\right)\right]+\mathrm{V}_{\mathrm{k}} \mathrm{V}_{\mathrm{cR}}\left[\mathrm{G}_{\mathrm{km}} \sin \left(\theta_{\mathrm{k}}-\delta_{\mathrm{cR}}\right)-\right. \\
& \left.\mathrm{B}_{\mathrm{km}} \cos \left(\theta_{\mathrm{k}}-\delta_{\mathrm{cR}}\right)\right]+\mathrm{V}_{\mathrm{k}} \mathrm{V}_{\mathrm{vR}}\left[\mathrm{G}_{\mathrm{vR}} \sin \left(\theta_{\mathrm{k}}-\delta_{\mathrm{vR}}\right)-\right. \\
& \left.\mathrm{B}_{\mathrm{vR}} \cos \left(\theta_{\mathrm{k}}-\delta_{\mathrm{vR}}\right)\right] \\
& \mathrm{P}_{\mathrm{m}}=\mathrm{V}_{\mathrm{m}}^{2} \mathrm{G}_{\mathrm{rm}}+\mathrm{V}_{\mathrm{k}} \mathrm{V}_{\mathrm{m}}\left[\mathrm{G}_{\mathrm{rk}} \cos \left(\theta_{\mathrm{m}}-\theta_{\mathrm{k}}\right)+\right. \\
& \left.B_{\mathrm{rk}} \sin \left(\theta_{\mathrm{m}}-\theta_{\mathrm{k}}\right)\right]+\mathrm{V}_{\mathrm{m}} \mathrm{V}_{\mathrm{cR}}\left[\mathrm{G}_{\mathrm{mm}} \cos \left(\theta_{\mathrm{m}}-\delta_{\mathrm{cR}}\right)+\right. \\
& \left.\mathrm{B}_{\mathrm{mm}} \sin \left(\theta_{\mathrm{m}}-\delta_{\mathrm{cR}}\right)\right] \\
& \mathrm{Q}_{\mathrm{m}}=-\mathrm{V}_{\mathrm{m}}^{2} \mathrm{G}_{\mathrm{rm}}+\mathrm{V}_{\mathrm{k}} \mathrm{V}_{\mathrm{m}}\left[\mathrm{G}_{\mathrm{rk}} \sin \left(\theta_{\mathrm{m}}-\theta_{\mathrm{k}}\right)-\right. \\
& \left.B_{\mathrm{rik}} \cos \left(\theta_{\mathrm{m}}-\theta_{\mathrm{k}}\right)\right]+\mathrm{V}_{\mathrm{m}} \mathrm{V}_{\mathrm{cR}}\left[\mathrm{G}_{\mathrm{rm}} \sin \left(\theta_{\mathrm{m}}-\delta_{\mathrm{cR}}\right)-\right. \\
& \left.\mathrm{B}_{\mathrm{m}} \cos \left(\theta_{\mathrm{m}}-\delta_{\mathrm{cR}}\right)\right] \\
& P_{\mathrm{cR}}=\mathrm{V}_{\mathrm{cR}}^{2} \mathrm{G}_{\mathrm{mm}}+\mathrm{V}_{\mathrm{cR}} \mathrm{V}_{\mathrm{k}}\left[\mathrm{G}_{\mathrm{km}} \cos \left(\delta_{\mathrm{cR}}-\theta_{\mathrm{k}}\right)+\right. \\
& \left.\mathrm{B}_{\mathrm{km}} \sin \left(\delta_{\mathrm{cR}}-\theta_{\mathrm{k}}\right)\right]+\mathrm{V}_{\mathrm{m}} \mathrm{V}_{\mathrm{cR}} \\
& {\left[\mathrm{G}_{\mathrm{mm}} \sin \left(\delta_{\mathrm{cR}}-\theta_{\mathrm{m}}\right)+\mathrm{B}_{\mathrm{mm}} \sin \left(\delta_{\mathrm{cR}}-\theta_{\mathrm{m}}\right)\right]} \\
& Q_{c R}=-V_{c R}^{2} B_{m m}+V_{c R} V_{k}\left[G_{k m} \cos \left(\delta_{c R}-\theta_{k}\right)-\right. \\
& \left.\mathrm{B}_{\mathrm{km}} \sin \left(\delta_{\mathrm{cR}}-\theta_{\mathrm{k}}\right)\right]+\mathrm{V}_{\mathrm{m}} \mathrm{V}_{\mathrm{cR}} \\
& {\left[\mathrm{G}_{\mathrm{mm}} \sin \left(\delta_{\mathrm{cR}}-\theta_{\mathrm{m}}\right)-\mathrm{B}_{\mathrm{mm}} \sin \left(\delta_{\mathrm{cR}}-\theta_{\mathrm{m}}\right)\right]} \\
& P_{\mathrm{vR}}=-\mathrm{V}_{\mathrm{vR}}^{2} \mathrm{G}_{\mathrm{vR}}+\mathrm{V}_{\mathrm{vR}} \mathrm{V}_{\mathrm{k}} \\
& {\left[\mathrm{G}_{\mathrm{vR}} \cos \left(\delta_{\mathrm{vR}}-\theta_{\mathrm{k}}\right)+\mathrm{B}_{\mathrm{vR}} \sin \left(\delta_{\mathrm{cR}}-\theta_{\mathrm{k}}\right)\right]} \\
& \mathrm{Q}_{\mathrm{vR}}=\mathrm{V}_{\mathrm{vR}}^{2} \mathrm{~B}_{\mathrm{vR}}+\mathrm{V}_{\mathrm{vR}} \mathrm{V}_{\mathrm{k}} \\
& {\left[\mathrm{G}_{\mathrm{vR}} \sin \left(\delta_{\mathrm{vR}}-\theta_{\mathrm{k}}\right)-\mathrm{B}_{\mathrm{vR}} \cos \left(\delta_{\mathrm{cR}}-\theta_{\mathrm{k}}\right)\right]}
\end{aligned}
$$

Assuming loss less converter valves, the active power supplied to the shunt converter, $\mathrm{P}_{\mathrm{vR}}$ equals the active power demanded by the series converter, $P_{C R}$ that is:

$$
\mathrm{P}_{\mathrm{vR}}+\mathrm{P}_{\mathrm{cR}}=0
$$

Furthermore if the coupling transformers are assumed to contain no resistance then, the active power at bus $\mathrm{k}$ matches the active power at bus $\mathrm{m}$, accordingly and we have:

$$
\mathrm{P}_{\mathrm{vR}}+\mathrm{P}_{\mathrm{cR}}=\mathrm{P}_{\mathrm{k}}+\mathrm{P}_{\mathrm{m}}=0
$$


The UPFC power equations in linearized form are combined with those of the $\mathrm{AC}$ network. For the case when the UPFC controls the following parameters: Voltage magnitude at the shunt converter terminal (bus k), active power flow from bus $\mathrm{m}$ to bus $\mathrm{k}$ and reactive power injected at bus $\mathrm{m}$.

Implemented model for optimizing location of the UPFC: Two types of UPFC model is represented in the studies. One is a coupled model and the other is decoupled model (Hingorani and Gyugyi, 1999). In the first type, a UPFC is modeled with a voltage source series with impedance in the transmission line. In the second type a UPFC is model with two separated buses.

The first type is more difficult compared with the second one and the modification of the Jacobean matrix of the system should be applied if it is used. On the other hand, the decoupled model can be easily used in conventional power flow methods without changing the Jacobean matrix of the system. In this study, the decoupled model (Fig. 3) is used for the power flow study. If the UPFC is assumed to be loss less, the real power flow $\mathrm{P}_{\mathrm{ij}}$ that flows from bus $\mathrm{i}$ to bus $\mathrm{j}$ can be written as:

$$
P_{i j}=P_{U 1}
$$

Although, a UPFC can control the power flow but cannot generate the real power. So Eq. 26 should be considered in the model:

$$
\mathrm{P}_{\mathrm{U} 1}+\mathrm{P}_{\mathrm{U} 2}=0
$$

The values of $\mathrm{Q}_{\mathrm{U} 1}, \mathrm{Q}_{\mathrm{U} 2}$ can be set to an arbitrary value within the capacity of the UPFC to maintain the bus voltage. In the same way if multiple UPFCs are installed in the power system, the control variables for the $\mathrm{kth}$ installed UPFC are shown as follows:

$$
\mathrm{UPFC}_{\mathrm{k}-\mathrm{th}}=\left[\mathrm{P}_{\mathrm{K} 1}^{\mathrm{U}}, \mathrm{Q}_{\mathrm{K} 1}^{\mathrm{U}}, \mathrm{P}_{\mathrm{K} 2}^{\mathrm{U}}, \mathrm{Q}_{\mathrm{K} 2}^{\mathrm{U}}\right]
$$

So that;

$$
\mathrm{P}_{\mathrm{K} 1}+\mathrm{P}_{\mathrm{K} 2}=0
$$

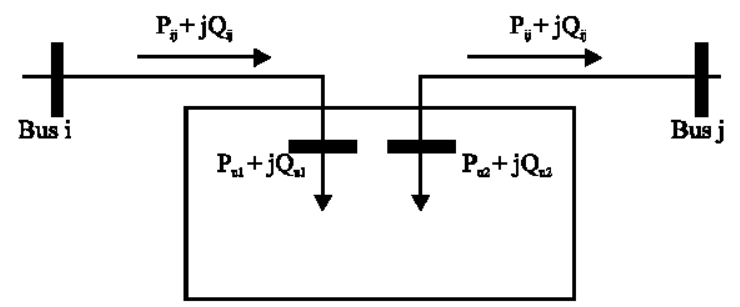

Fig. 3: The decoupled model of UPFC

\section{Optimization algorithm and implementation}

HGAPSO algorithm: The genetic algorithm can handle any kind of objective functions and constraints without much mathematical requirements about the optimization problems. GA has been touted as a class of generalpurpose search strategies for optimization problems. In GA, variables of a problem are represented as genes in a chromosome and the chromosomes are evaluated according to their fitness values. GA starts with a set of randomly selected chromosomes as the initial population that encodes a set of possible solutions.

Through natural selection and genetic operators, mutation and crossover chromosomes with better fitness are found. The genetic operators alter the composition of genes to create new chromosomes called offspring. The selection operator is an artificial version of the natural selection, a Darwinian survival of the fittest among the population to create populations from generation to generation and chromosomes with better fitness have higher probabilities of being selected in the next generation (Patemi et al., 1999; Coley, 1999).

The PSO method is a population-based one and is described by its developers as an optimization paradigm which models the social behavior of birds flocking or fish schooling for food. Therefore, PSO works with a population of potential solutions rather than with a single individual (Ratnaweera et al., 2004). It has also been found to be robust in solving problem featuring non-linear, non-differentiable and high-dimensional (Shayeghi et al., 2009). The PSO starts with a population of random solutions particles in a D-dimension space. The ith particle is represented by $\mathrm{X}_{\mathrm{i}}=\left(\mathrm{x}_{\mathrm{i} 1}, \mathrm{x}_{\mathrm{i} 2}, \ldots, \mathrm{X}_{\mathrm{iD}}\right)$. Each particle keeps track of its coordinates in hyperspace which are associated with the fittest solution, it has achieved so far. The value of the fitness for particle $i$ (pbest) is also stored as $\mathrm{P}_{\mathrm{i}}=\left(\mathrm{p}_{\mathrm{i} 1}, \mathrm{p}_{\mathrm{i} 2}, \ldots, \mathrm{p}_{\mathrm{iD}}\right)$. The global version of the PSO keeps track of the overall best value (gbest) and its location, obtained thus far by any particle in the population. The PSO consists of at each step, changing the velocity of each particle toward its pbest and gbest according to Eq. 29. The velocity of particle $i$ is represented as $V_{i}=\left(v_{i 1}, v_{i 2} \ldots v_{i D}\right)$. Acceleration is weighted by a random term with separate random numbers being generated for acceleration toward pbest and gbest. The position of the ith particle is then updated according to Eq. 30 (Shayeghi et al., 2009):

$$
\begin{aligned}
\mathrm{v}_{\mathrm{id}}= & \mathrm{k} \times \mathrm{v}_{\mathrm{id}}+\mathrm{c}_{1} \times \operatorname{ran}(\mathrm{d}) \times\left(\mathrm{P}_{\mathrm{id}}-\mathrm{x}_{\mathrm{id}}\right)+ \\
& \mathrm{c}_{2} \times \operatorname{ran}(\mathrm{d}) \times\left(\mathrm{P}_{\mathrm{gd}}-\mathrm{x}_{\mathrm{id}}\right) \\
& \mathrm{x}_{\text {in }}(\mathrm{t}+1)=\mathrm{x}_{\text {in }}(\mathrm{t})+\mathrm{v}_{\text {in }}(\mathrm{t}+1)
\end{aligned}
$$


where, $\mathrm{P}_{\text {id }}$ and $\mathrm{P}_{\text {gd }}$ are pbest and gbest. The positive constants $\mathrm{c}_{1}$ and $\mathrm{c}_{2}$ are the cognitive and social components that are the acceleration constants responsible for varying the particle velocity towards pbest and gbest, respectively. Variables $r_{1}$ and $r_{2}$ are two random functions based on uniform probability distribution functions in the range $(0,1)$.

The use of variable $\mathrm{w}$ is responsible for dynamically adjusting the velocity of the particles so it is responsible for balancing between local and global searches hence, requiring less iteration for the algorithm to converge (Poli et al., 2007). The considered optimization problem can be solved by either particle swarm intelligence or genetic algorithm. A combination of GA and PSO is utilized to overcome the drawbacks of using each of them solely, named HGAPSO optimization algorithm. Genetic algorithm has the capability of global optimum finding but low convergence speed near global optimum on the contrary, PSO has high convergence speed but the probability of trapping on the local optimum. In the proposed algorithm, individuals are coded to a chromosome that contains variables of the problem.

The configuration of chromosome in order to find optimal location of the UPFC consists of two types of parameters: location of UPFC and $P_{i}, Q_{i}, P_{j}$ and $Q_{j}$ as decoupled model parameters of the UPFC. In Fig. 4, the chromosome for the proposed algorithm has been shown (Gerbex et al., 2001). The aim of the optimization is to perform a best utilization of the existing transmission lines. In this respect, UPFC device is located in order to maximize the system loadability while observing thermal and voltage constraints. In other words, it was tried to increase the power transmitted by power system as much as possible to the costumers with holding power system in security state in terms of branch loading and voltage levels.

The objective function is made in order to penalize configurations of the UPFC which lead to overload transmissions lines and over or under voltage at busses. The objective function is defined as the sum of two terms. The first one is related to the branch loading which penalizes overloads in lines. This term is called LF and computed for all lines of the power system if branch loading is $<100 \%$ its value is equal to 1 ; otherwise it decreases exponentionally with respect to the overload. To accelerate the convergence, product of values for all

\begin{tabular}{|l|c|c|c|c|c|}
\hline $\begin{array}{l}\text { Objective } \\
\text { function }\end{array}$ & $\begin{array}{c}\text { Location of the } \\
\text { UPFC }\end{array}$ & $\mathbf{P}_{\mathrm{i}}$ & $\mathbf{Q}$ & $\mathbf{P}_{\mathrm{j}}$ & $\mathbf{Q}$ \\
\hline
\end{tabular}

Fig. 4: The chromosome of the proposed algorithm objective functions is calculated. The second part of of the objective functions is equal to 1 . Outside this range, value decreases exponentionally with the voltage the objective function is for voltage levels that are named $\mathrm{BF}$. This function is calculated for all buses of power system. For voltage levels between 0.95 and 1.05 , values deviations. Therefore for a configuration of UPFCs, objective function is given as:

$$
\begin{gathered}
\mathrm{LF}=\left\{\begin{array}{ll}
1, & \mathrm{BL}<100 \\
\exp [0.0461(100-\mathrm{BL})], & \mathrm{BL} \geq 100
\end{array}\right\} \\
\mathrm{BF}=\left\{\begin{array}{ll}
1, & 0 \leq \mathrm{V}_{\mathrm{L}} \leq 100 \\
\exp \left[-23.0259\left|1-\mathrm{V}_{\mathrm{L}}\right|-0.05\right] & , .05 \leq \mathrm{V}_{\mathrm{L}} \leq 1.25 \\
& \text { or } 0.75 \leq \mathrm{V}_{\mathrm{L}} \leq 0.95
\end{array}\right\} \\
\text { Objective function of }=\prod_{\mathrm{i}=\text { line }} \mathrm{LF}_{\mathrm{i}}+\prod_{\mathrm{j}=\text { buses }} \mathrm{BF}_{\mathrm{i}}
\end{gathered}
$$

Where:

$\mathrm{LF}=$ The Line Flow index

$\mathrm{BL}=$ The Branch Loading (percentage of the line flow with respect to the line capacity rate)

$\mathrm{BF}=$ Bus voltage index

$\mathrm{V}_{\mathrm{L}}=$ Per unit value of the bus voltages

Optimization strategy: The hybrid GA and PSO are applied to conduct searching optimum for the parameter set of the power system stability. The optimization process is given as:

Step 1: Define the varying range of the parameters and objective function over the parameters.

Step 2: Set generation gen $=0$.

Step 3: Initialize population of the GA and particles of the PSO.

Step 4: Evaluate population of GA and particles of PSO.

Step 5: Perform selection operator of the GA.

Step 6: Perform crossover and mutation operators of the GA.

Step 7: Modify each particle's searching point by Eq. 29 and 30 .

Step 8: Evaluate new population of the GA and new particles of the PSO.

Step 9: If the termination criterion has satisfied then stop; otherwise, gen $=$ gen +1 and go to step 5 . 
For the initialization of GA, the initial population $\mathrm{P}$ is generated randomly and chromosomes encoded as the parameter set are encoded into binary string and the mapping from a binary string to a real number (r) is calculated as follows:

$$
\mathrm{r}=\min _{\mathrm{r}}+\text { binrep } \times \frac{\max _{\mathrm{r}}-\min _{\mathrm{r}}}{2^{1}-1}
$$

Where, $\min _{\mathrm{r}}$ is the minimum value of the input variable, max the maximum value of the input variable and binrep represents the decimal value of length 1 . The traditional roulette selection with elitism is performed as the selection operator and it ensures that the best chromosome is selected into the new generation for the GA. The two point crossover will act on parents to generate offspring. Mutation is keeping diversity in the population for the PSO. The initial particles $\mathrm{p} \in\left[\mathrm{min}_{\mathrm{r}}, \max _{\mathrm{r}}\right]$ are randomly generated and new particles are created by Eq. 29 and 30 . The inertia weight, $\mathrm{k}$ is given by:

$$
\mathrm{k}=\left(\mathrm{k}_{1}-\mathrm{k}_{2}\right) \times \frac{(\text { MAXGEN }- \text { gen })}{\text { MAXGEN }}-\mathrm{k}_{2}
$$

Where, $\mathrm{K}_{1}$ and $\mathrm{K}_{2}$ are the initial and final value of weight, respectively; gen is the current generation number and MAXGEN is the maximum number of generation. The fitness for the GA and PSO is computed by maximizing the inverse of the overshoot defined as follows:

$$
\text { Fitness }=\frac{1}{\mathrm{OF}}=\frac{1}{\prod_{\mathrm{i}=\text { line }} \mathrm{LF}_{\mathrm{i}}+\prod_{\mathrm{j}=\text { buses }} \mathrm{BF}_{\mathrm{i}}}
$$

In the implementation, the size of the initial population for GA and PSO is 100. The crossover probability $P_{c}$, mutation Probability $P_{m}=$ MAXGEN $=100$, $\mathrm{c}_{1}=1, \mathrm{c}_{2}=1, \mathrm{k}_{1}=0.9, \mathrm{k}_{2}=0.4,1=10$.

Case study: The load increasing studies on the real power system are done for different aims in planning and operation process of the system. For long term studies of the power system, it is necessary to consider both active and reactive load increasing. The standard IEEE 14 bus test system is shown in Fig. 5 to demonstrate the effectiveness and validity of the proposed method. The numerical data and parameters are taken from Gerbex et al. (2001). In this way, new values of voltages and active and reactive powers of the net and also the value of the objective function are obtained. By iterating this process and a comparison between fitness values, the best chromosome meaning best found solution is introduced. Suppose that the given number of UPFCs is set to 2. The dynamic optimization performance of the solution method is shown in Fig. 6. In case of using

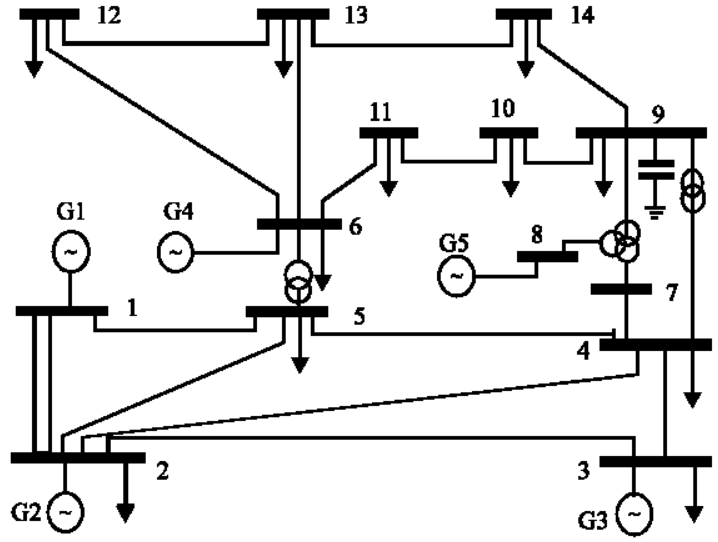

Fig. 5: A standard IEEE 14 bus system

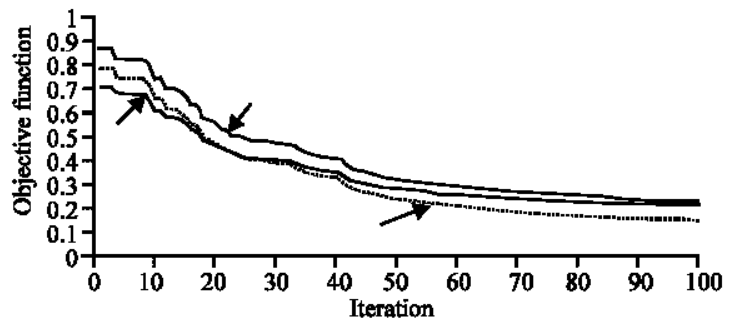

Fig. 6: Dynamic optimization performance

\begin{tabular}{|c|c|c|c|c|c|}
\hline Bus no. & $P_{i}$ & $\mathrm{Q}_{j}$ & $P_{j}$ & $Q_{j}$ & Fitness \\
\hline 3 & -132.0 & 97.00 & -205.00 & -143.60 & 0.1201 \\
\hline 4 & -11.4 & -2.24 & 15.87 & 82.66 & - \\
\hline 5 & - & - & - & - & - \\
\hline 6 & - & - & - & - & - \\
\hline
\end{tabular}

Table 1: The best chromosome for 1 UPFC
\begin{tabular}{llllll} 
Bus no. & \multicolumn{1}{l}{$\mathrm{P}_{\mathrm{i}}$} & $\mathrm{Q}_{\mathrm{j}}$ & \multicolumn{1}{c}{$\mathrm{P}_{j}$} & $\mathrm{Q}_{j}$ & Fitness \\
\hline 9 & -41.67 & -23.81 & -204.9 & -145.6 & 0.0593 \\
4 & - & - & - & - & - \\
\hline
\end{tabular}

Table 2: The best chromosome for 2 UPFC

just one UPFC in the system, the proposed algorithm yields a 7 segmented chromosome as the optimal solution. In Table 1, the numbers one and four express the bus number which one UPFC is located between them. The second and third numbers show the values of injective active and reactive powers, respectively into the bus 9 and the 5 th and 6 th numbers, respectively show the injective active and reactive powers into the bus 4 . 7 th number also gives the fitness value defined in the Eq. 36 for the UPFC.In case of using two UPFC in the power system simultaneously, the proposed algorithm represents the optimum solution by 13 segmented chromosome. The first UPFC is set between the buses number 3 and 4 and the second UPFC is set between the buses number 5 and 6 . The simulation result is shown in Table 2. The numerical results before and after UPFC placement in the network is shown in Table 3-11 and the corresponding diagrams are also shown in Fig. 7-9. It is observed form the simulation results that 
Int. J. Elec. Power Eng., 4 (3): 169-176, 2010

Table 3: The result of buses voltages of power flow without UPFC

\begin{tabular}{lccl}
\hline Bus no. & Voltage & Bus no. & Voltage \\
\hline 1 & 1.050 & 8 & 1.050 \\
2 & 1.050 & 9 & 0.901 \\
3 & 1.036 & 10 & 0.888 \\
4 & 0.985 & 11 & 0.957 \\
5 & 1.000 & 12 & 0.947 \\
6 & 1.050 & 13 & 0.953 \\
7 & 0.955 & 14 & 0.859 \\
\hline
\end{tabular}

Table 4: The result of buses voltages of power flow wiht 1 UPFC

\begin{tabular}{llcc}
\hline Bus no. & Voltage & Bus no. & Voltage \\
\hline 1 & 1.050 & 8 & 1.050 \\
2 & 1.050 & 9 & 1.051 \\
3 & 1.011 & 10 & 0.970 \\
4 & 1.023 & 11 & 1.008 \\
5 & 1.032 & 12 & 1.015 \\
6 & 1.048 & 13 & 1.023 \\
7 & 0.981 & 14 & 0.987 \\
\hline
\end{tabular}

Table 5: The result of buses voltages of power flow with 2 UPFC

\begin{tabular}{lccc} 
Bus no. & Voltage & Bus no. & Voltage \\
\hline 1 & 1.050 & 8 & 1.050 \\
2 & 1.050 & 9 & 1.068 \\
3 & 1.011 & 10 & 0.999 \\
4 & 1.005 & 11 & 1.002 \\
5 & 1.014 & 12 & 0.995 \\
6 & 1.048 & 13 & 1.006 \\
7 & 1.005 & 14 & 0.998 \\
\hline
\end{tabular}

Table 6: The result of active power flow without UPFC

\begin{tabular}{lccc}
\hline Line no. & Power flow & Line no. & Power flow \\
\hline 1 & 0.907 & 11 & -3.710 \\
2 & 0.123 & 12 & -1.290 \\
3 & 0.035 & 13 & -0.020 \\
4 & 0.288 & 14 & 1.621 \\
5 & 0.170 & 15 & 1.494 \\
6 & 0.115 & 16 & 1.278 \\
7 & 0.014 & 17 & 0.093 \\
8 & 0.086 & 18 & 0.739 \\
9 & -0.120 & 19 & 0.420 \\
10 & 0.420 & 20 & 0.002 \\
\hline
\end{tabular}

Table 7: The result of reactive power flow without UPFC

\begin{tabular}{lccc}
\hline Line no. & Power flow & Line no. & Power flow \\
\hline 1 & 0.406 & 11 & -0.560 \\
2 & 0.055 & 12 & 0.286 \\
3 & 0.028 & 13 & 0.655 \\
4 & 0.186 & 14 & 0.443 \\
5 & 0.207 & 15 & 0.344 \\
6 & 0.169 & 16 & 0.539 \\
7 & -0.080 & 17 & -0.060 \\
8 & -0.030 & 18 & -0.160 \\
9 & -0.100 & 19 & -0.280 \\
10 & 0.210 & 20 & 0.613 \\
\hline
\end{tabular}

Table 8: The result of active power flow with 1 UPFC

\begin{tabular}{lccc} 
Line no. & Power flow & Line no. & Power flow \\
\hline 1 & 0.467 & 11 & -0.950 \\
2 & 0.141 & 12 & -0.980 \\
3 & 0.053 & 13 & 0.163 \\
4 & 0.340 & 14 & 0.894 \\
5 & 0.257 & 15 & 1.019 \\
6 & 0.187 & 16 & 1.059 \\
7 & -0.040 & 17 & 0.178 \\
8 & 0.136 & 18 & 0.895 \\
9 & -0.170 & 19 & 0.626 \\
10 & 0.326 & 20 & 0.003 \\
\hline
\end{tabular}

Table 9: The result of reactive power flow with 1 UPFC

\begin{tabular}{lccc}
\hline Line no. & Power flow & Line no. & Power flow \\
\hline 1 & 0.589 & 11 & -0.450 \\
2 & 0.055 & 12 & 0.254 \\
3 & 0.028 & 13 & 0.711 \\
4 & 0.188 & 14 & 0.566 \\
5 & 0.210 & 15 & 0.222 \\
6 & 0.170 & 16 & 0.583 \\
7 & -0.080 & 17 & -0.060 \\
8 & -0.030 & 18 & -0.180 \\
9 & -0.100 & 19 & -0.290 \\
10 & 0.214 & 20 & 0.624 \\
\hline
\end{tabular}

Table 10: The result of active power flow with 2 UPFC

\begin{tabular}{lccc}
\hline Line no. & Power flow & Line no. & Power flow \\
\hline 1 & 0.695 & 11 & -0.900 \\
2 & 0.043 & 12 & -0.830 \\
3 & 0.086 & 13 & 0.180 \\
4 & 0.410 & 14 & 1.123 \\
5 & 0.186 & 15 & 1.092 \\
6 & 0.191 & 16 & 1.133 \\
7 & -0.010 & 17 & 0.091 \\
8 & 0.195 & 18 & 0.947 \\
9 & -0.680 & 19 & 0.715 \\
10 & 0.415 & 20 & 0.001 \\
\hline
\end{tabular}

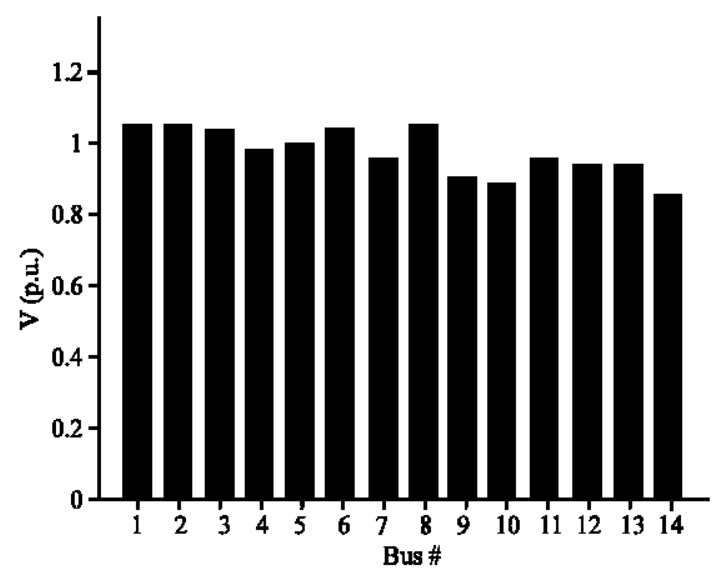

Fig. 7: Voltage profile without using UPFC

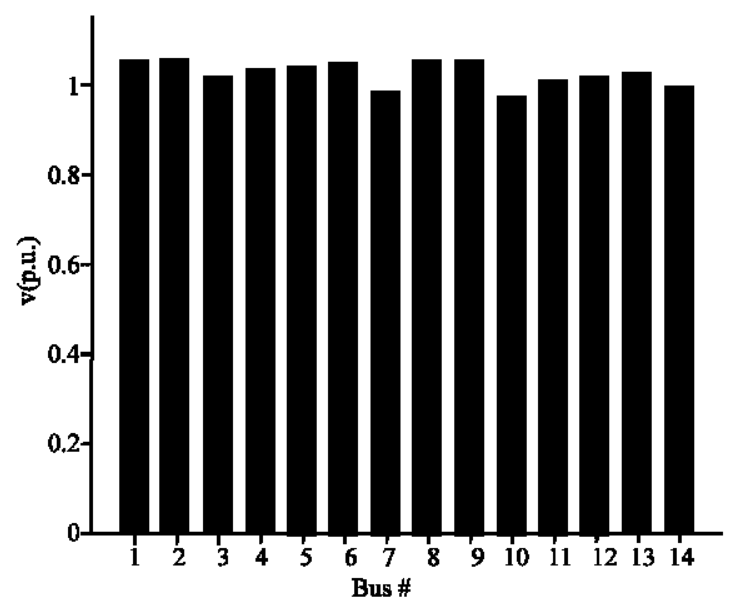

Fig. 8: Voltage profile with using 1 UPFC 


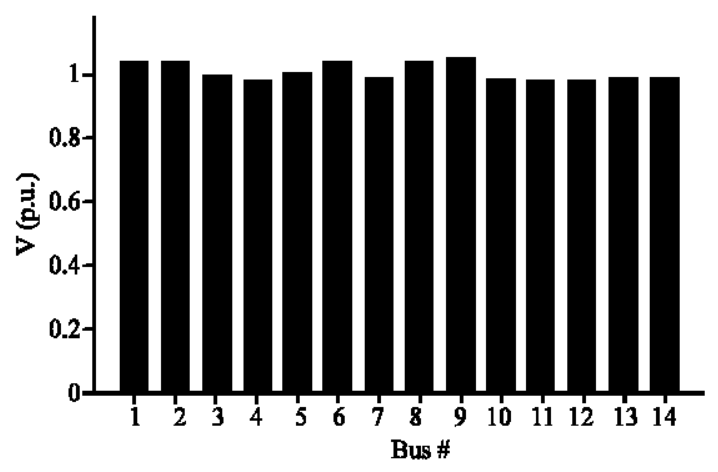

Fig. 9: Voltage profile with using 2 UPFC

\begin{tabular}{|c|c|c|c|}
\hline Line no. & Power flow & Line no. & Power flow \\
\hline 1 & 0.495 & 11 & -0.610 \\
\hline 2 & 0.278 & 12 & 0.254 \\
\hline 3 & -0.010 & 13 & 0.711 \\
\hline 4 & 0.188 & 14 & 0.596 \\
\hline 5 & 0.188 & 15 & 0.222 \\
\hline 6 & 0.150 & 16 & 0.583 \\
\hline 7 & -0.060 & 17 & -0.060 \\
\hline 8 & -0.060 & 18 & -0.180 \\
\hline 9 & -0.590 & 19 & -0.290 \\
\hline 10 & -0.206 & 20 & 0.624 \\
\hline
\end{tabular}

the voltages of buses of the network are in authorized range and the profile of the voltage is satisfactorily flat.

\section{CONCLUSION}

In this study, the optimal UPFC placement on an unstable power system because of load increasing has been investigated. A mathematical model for simultaneously optimizing location and parameters of the UPFCs is presented in this study. A hybrid genetic and particle swarm optimization algorithms is used to solve this nonlinear programming problem. The computation process are discussed in detail such as the construction of the chromosome, handling of equality and inequality constraints, the location of UPFC to be embedded and the load flow computation etc. The case study of the IEEE 14 busbar system has confirmed that the developed algorithm is correct and effective.

\section{RECOMMENDATION}

The results of the simulations suggest a further use of the proposed tool in the planning field. With the proposed methodology, it can be seen that the FACTS device candidate should give the best locations with respect to various future load duration curves of the transmission system. Such information is of strategic importance to analyze the variation of the system parameters with respect to the number and optimal placement of the UPFC and consequently with respect to the increase of the power transfers.

\section{REFERENCES}

Coley, D.A., 1999. An Introduction to Genetic Algorithms for Scientists and Engineers. 1st Edn., World Scientific Press, Singapore.

Fang, W.L. and H.W. Ngan, 1999. Optimizing location of unified power flow controllers using the method of augmented Lagrange multipliers. IEEE Proc. Gener. Trans. Dist., 146: 428-434.

Gerbex, S., R. Cherkaoui and J.A. Germond, 2001. Optimal location of multi-type FACTS devices in a power system by means of genetic algorithms. Int. J. Electrical Power Energy Syst., 16: 537-544.

Hao, J., L.B. Shi and C. Chen, 2004. Optimising location of unified power flow controllers by means of improved evolutionary programming. IEEE Proc. Gen. Trans. Dist., 151: 705-712.

Hingorani, N.G. and L. Gyugyi, 1999. Understanding FACTS: Concepts and Technology of Flexible AC Transmission System. 1st Edn., IEEE Press, New York.

Nabavi-Niaki, A. and M.R. Iravani, 1996. Steady-state and dynamic models of unified power flow controller (UPFC) for power system studies. IEEE Trans. Power Syst., 11: 1937-1943.

Noroozian, M., L. Angquist, M. Ghandhari and G. Andersson, 1997. Use of UPFC for optimal power flow control. IEEE Trans. Power Delivery, 12: 1629-1634.

Patemi, P., S. Vitet, M. Bena and A. Yokoyama, 1999. Optimal location of phase shifters in French network by genetic algorithm. IEEE Trans. Power Syst., 14: 37-42.

Poli, R., J. Kennedy and T. Blackwell, 2007. Particle swarm optimization: An overview. Swarm Intell., 1: 33-57.

Ratnaweera, A., S.K. Halgamuge and H.C. Watson, 2004. Self organizing hierarchical particle swarm optimizer with time varying acceleration coefficients. IEEE Trans. Evol. Comput., 8: 240-255.

Shayeghi, H., H.A. Shayanfar, S. Jalilzadeh and A. Safari, 2009. A PSO based unified power flow controller for damping of power system oscillations. Energy Conversion Manage., 50: 2583-2592.

Singh, S.N. and A.K. David, 2001. Optimal location of FACTS devices for congestion management. Electr. Power Syst. Res., 58: 71-79. 